Goldschmidt 2021 Abstract

https://doi.org/10.7185/gold2021.7379

\section{Assessing the analytical potential of optical tweezers for sample return missions}

ANNA MUSOLINO ${ }^{1}$, ALESSANDRO MAGAZZU' ${ }^{2}$, DAVID BRONTE CIRIZA $^{2}$, MARTIN DAVID SUTTLE ${ }^{3,4}$, ALESSANDRA ROTUNDI ${ }^{5}$, JOHN ROBERT BRUCATO ${ }^{6}$, PAOLO POLIMENO $^{2,7}$, ROSALBA SAIJA ${ }^{7}$, MARIA ANTONIA IATİ ${ }^{2}$, ANTONINO FOTI ${ }^{2}$, MARIA DONATO ${ }^{2}$, PIETRO GIUSEPPE GUCCIARDI ${ }^{2}$, ONOFRIO M. MARAGÒ ${ }^{2}$ AND LUIGI FOLCO ${ }^{8,9}$

${ }^{1}$ Università di Pisa

${ }^{2} \mathrm{CNR}$-IPCF, Istituto per i Processi Chimico-Fisici, Messina

${ }^{3}$ Dipartimento di Scienze della Terra, Università di Pisa

${ }^{4}$ Planetary Materials Group, The Natural History Museum, London

${ }^{5}$ Dipartimento di Scienze e Tecnologie, Università di Napoli "Parthenope", Napoli

${ }^{6}$ INAF, Osservatorio di Astrofisica di Arcetri, Firenze

${ }^{7}$ Dipartimento di Scienze Matematiche e Informatiche, Scienze Fisiche e Scienze della Terra, Università di Messina

${ }^{8}$ Dipartimento di Scienze della Terra, Università di Pisa, Pisa ${ }^{9}$ CISUP, Università di Pisa

Presenting Author: a.musolino@studenti.unipi.it

Optical tweezers are novel tools based on highly focused laser beams enabling contactless, non-invasive trapping and manipulation of particulate matter in different media like liquids, air and vacuum. When coupled with Raman spectrometers (Figure 1), optical tweezers allow high-resolution (i.e., no background noise from substrate) spectroscopic analysis of physico-chemical properties. Although in recent years they have been widely and successfully used in a variety of research fields [1], their application in space science is in its infancy [2, 3].

In principle, optical tweezers have key prerequisites for maximizing the scientific return from the analyses of planetary dust samples collected by the current and future sample return missions (e.g. Hayabusa-2, OSIRIS-Rex, Mars 2020), particularly during the preliminary investigation procedures in receiving/curatorial facilities. High-resolution, contactless and non-invasive analyses of planetary dust are actually expected to i) provide unprecedented information on the astrophysical origin and geologic evolution of their parent bodies and ii) be instrumental for biohazard assessment for constrained sample return missions - like those targeting Mars or icy bodies in the outer solar system, and the detection of past or extant extraterrestrial life.

Optical tweezers, developed in our laboratories [4], were used to collect individual dust grains from powdered analogues and extraterrestrial materials of known composition and to determine their composition and response to optical forces without substrate effects. Results (e.g., Figure 2) document the high potential of this novel technique and will be used to assess its effectiveness in the micromanipulation and analysis of extraterrestrial dust from sample return missions in receiving/curatorial facilities and its potential role within coordinated multiscale analytical protocols.

Acknowledgements. ASI-INAF, "SPACE Tweezers", PI\# I/024/12/0 2018-16-HH.0; PNRA, "Meteoriti Antartiche", PI\# PNRA160029, EC- Horizon 2020 “ActiveMatter”, PI\# 812780.

References

[1] Jones et al., (2015) "Optical tweezers: Principles and applications", CUP.

[2] Alali et al., (2020) J. Quant. Spectr. Rad. Trans., 255, 107249.

[3] Polimeno et al., (2021) EPJ plus (under rev).

[4] Gillibert et al., (2019) Environ. Sci. Technol., 53, $9003-$ 9013.

[5] Collareta et al., (2016) MAPS, 51, 351-371 (2016).

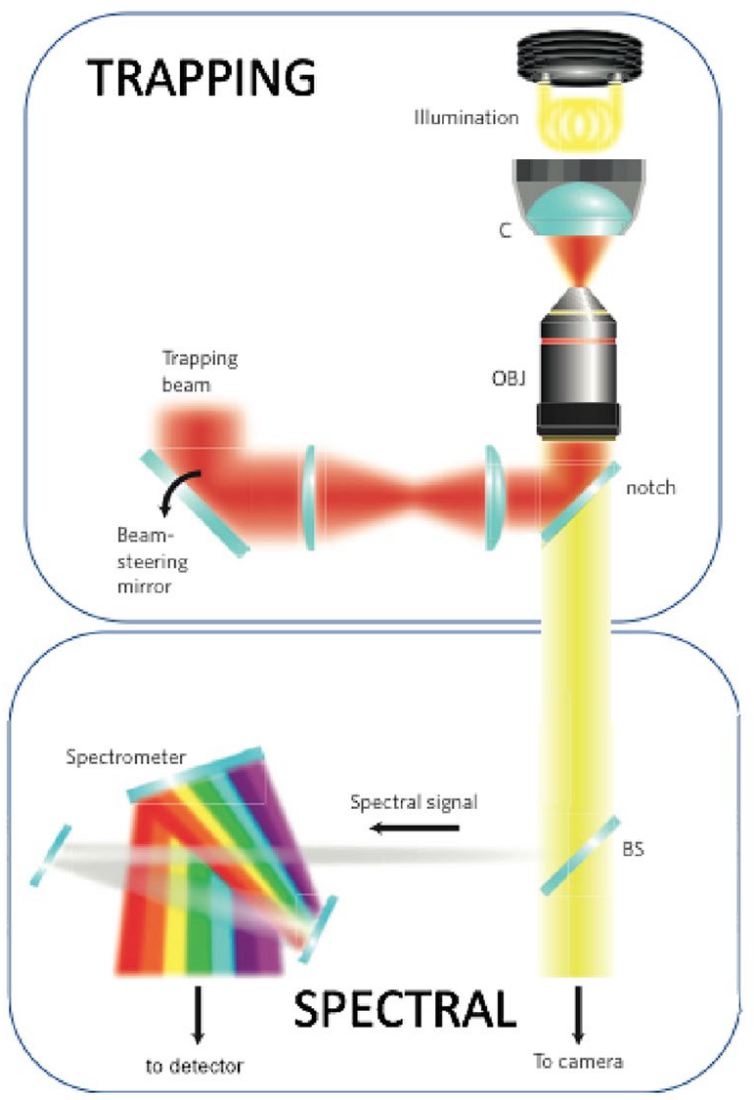

Figure 1: Sketch of the optical tweezers setup with Raman spectroscopy integration.

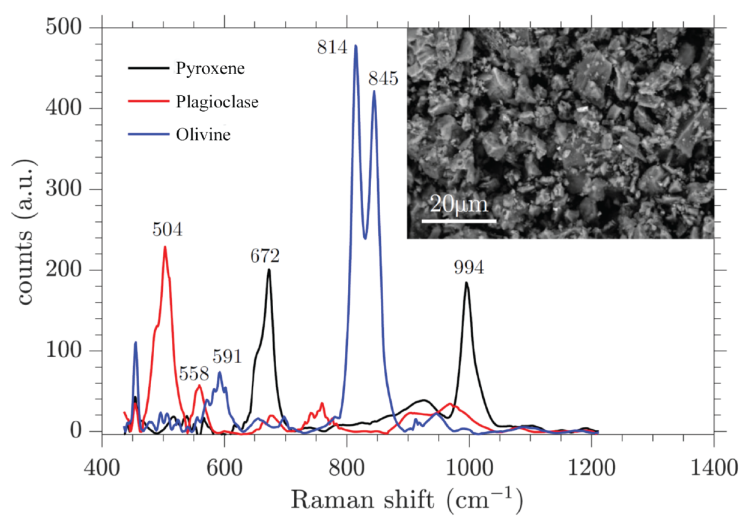

Figure 2: Raman spectra of optically trapped micron-sized fragments from the DEW 12007 meteorite (lunar regolith breccia) showing a good match with the expected mineral composition from literature [5]. 